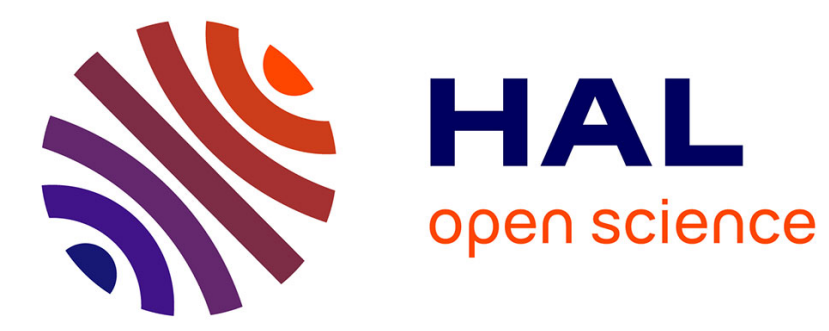

\title{
Some implications of common consequences in lotteries
}

David Crainich, Louis Eeckhoudt, Mario Menegatti

\section{To cite this version:}

David Crainich, Louis Eeckhoudt, Mario Menegatti. Some implications of common consequences in lotteries. Journal of Risk and Uncertainty, 2019, 59, pp.185-202. 10.1007/s11166-019-09314-4 . hal-02502811

\section{HAL Id: hal-02502811 \\ https://hal.science/hal-02502811}

Submitted on 20 Oct 2021

HAL is a multi-disciplinary open access archive for the deposit and dissemination of scientific research documents, whether they are published or not. The documents may come from teaching and research institutions in France or abroad, or from public or private research centers.
L'archive ouverte pluridisciplinaire HAL, est destinée au dépôt et à la diffusion de documents scientifiques de niveau recherche, publiés ou non, émanant des établissements d'enseignement et de recherche français ou étrangers, des laboratoires publics ou privés. 


\title{
Some implications of common consequences in lotteries
}

\author{
DAVID CRAINICH \\ CNRS (LEM, UMR 9221) and ISEG School of Management \\ 3, rue de la Digue \\ 59000 Lille, France \\ Tel: +3320545892 \\ Email: d.crainich@ieseg.fr \\ LOUIS EECKHOUDT \\ IESEG School of Management \\ 3 , rue de la Digue \\ 59000 Lille, France \\ Tel: +3320545892 \\ Email: louis.eeckhoudt@fucam.ac.be

\section{MARIO MENEGATTI} \\ Dipartimento di Scienze Economiche e Aziendali \\ Università degli Studi di Parma \\ via J.F. Kennedy 6 \\ 43125 Parma, Italy \\ Tel: +390521032450 \\ Email: mario.menegatti@unipr.it
}




\begin{abstract}
This work studies the implications of some aspects of preferences toward risk in the choice between two binary lotteries exhibiting a common consequence. The results obtained are then applied to two different problems: the choice between two risky challenges characterized by different rewards in the case of success and different probabilities of success and the choice between self-protection and selfinsurance in the presence of the risk of incurring financial loss.
\end{abstract}

JEL Classification: D81

Keywords: lotteries; risk aversion; risky challenges; self-protection; self-insurance 


\section{Introduction}

There are many instances in life where individuals or organizations undertake an action, which, in the case of success, will improve their status. Many examples can be provided. A student may wish to obtain a university degree or apply for a grant. A firm may invest in a new production line or apply for public funding. A researcher may start a new project with the aim of solving a problem or inventing a new product. An individual may take part in a sports competition or place a bet on it. In all these cases, agents face a "challenge", where they can succeed and thus improve their status, or fail and leave their status unchanged.

Such challenges have various facets. In particular, there may be more than one possibility, so that a relevant question may be which of the possible challenges the agent chooses to take up. In the case where the agent has to choose between different challenges a specific but very significant question can be asked: Does the agent prefer to face a challenge where the possible reward is high but probability of success is low ("hard challenge") or a challenge where the reward is lower but the probability of success is higher ("soft challenge")? Or, alternatively, what type of agent prefers the hard challenge and what type of agent prefers the soft challenge?

In fact, when a firm compares different choices for new production lines or different kinds of public funding, some will have higher returns in the case of success, but others will have a higher chance of success. Comparing different universities, a student may choose a more selective institution where succeeding ensures a better status but probability of failure is higher, or a less selective institution where it is easier to succeed but the degree obtained is less prestigious. In a sports competition, an individual can take part in an international championship with high reward but strong competitors, or in a local tournament with lower reward but weaker competitors. And placing bets, people may choose to bet on a more probable event for a lower reward, or on a less probable event for a higher reward. ${ }^{1}$

\footnotetext{
${ }^{1}$ We emphazise that this idea of challenge, although significatly different, is potentially related to the concept of contests between agents, first introduced by Tullock (1980) and Dixit (1987) and more recently extended to the case of risk averse agents (e.g. Konrad and Schlesinger, 1997; Treich, 2010 and Liu et al. 2018). This literature considers many agents competing in a contest where reward is a kind of rent. Our framework examines instead the optimal choice of a single agent, who has to choose between different challenges whose probability of success is given. Moreover, the goal of the contest models is the study of the level of effort in a (Nash) equilibrium, while our aim is the analysis of the role of the attitude toward risk in the choice between challenges. All this suggests a
} 
Considering a context completely different from that described above, there are many situations where an individual faces the risk of incurring a future financial loss. Many examples of this kind of situation can be provided. The owner of a property may face the risk of fire damage. A driver may face the risk of a car accident. The belongings of an agent may be subject to the risk of burglary.

When facing these situations, an agent can act in different ways. In particular, ignoring the instrument of market insurance, the agent can implement many activities in order to deal with the risk of financial loss. One strategy may be to generate a reduction in the probability that the loss occurs. Acting in this direction, the owner of a property can improve the safety of the electrical system in order to reduce the probability of a fire accident. Similarly, a driver can make periodical maintenance of his car in order to reduce the probability of accidents. Lastly, the installation of an alarm may reduce the probability of burglary.

The same risks, however, may be dealt with by means of the different strategy of reducing the size of the possible loss, leaving the probability that it occurs unchanged. Acting in this direction, the owner of a property can reduce the damages in case of a fire accident by installing a fire extinguishing system. A driver can reduce the negative effects of a car accident by fastening the safety belt. The loss in case of a burglary may be reduced if some valuables are put in a safe-deposit box.

The two instruments used to deal with the risk of incurring a loss described above are typically called in risk theory "self-protection" and "self-insurance". 2 These two instruments may be used together (as, for instance, in the case of both maintenance and safety belt in the example of car accident risk) but, in some cases, an agent has to choose between them because of budget constraints, time constraints or other kinds of constraints. For instance, because of budget constraints, the owner of a property may be forced to choose between installing a new electrical system and a fire extinguishing system or an agent may have to choose between installing an alarm and paying the rent of a safe-deposit box.

The issue of the choice between risky challenges and the issue of the choice between self-protection and self-insurance are different problems, but they share an important possible complementarity between the approach in the literature studying contests and our approach in considering the choice between risky challenges.

${ }^{2}$ This terminology derives from the seminal work by Ehrlich and Becker (1972), which started a wide literature on these issues (e.g. Dionne and Eeckhoudt, 1985; Snow, 2011 and Hoffman and Peter, 2016). 
element: both problems are described by lotteries exhibiting a kind of "common consequence" in the two choices. In particular, when an agent compares two different challenges, he compares two different binary lotteries, where the two possible events are "success" and "failure". The probability of success is different in the two lotteries and, in the case of success, the agent gains a reward which is also different. On the other hand, in the case of failure, agent wealth is unchanged, and it is thus the same in the two lotteries. Similarly, when an agent compares self-protection and self-insurance, he compares two lotteries where the two possible events are "loss occurrence" and "no loss". The probability of loss occurrence is different in the two lotteries and the size of the loss in case of occurrence is different too. On the other hand, if the loss does not occur, agent wealth is unchanged and it is thus the same in the two lotteries.

The existence of a common consequence, which is present both in the choice between risky challenges (in the case of "failure") and in the choice between self-protection and and self-insurance (in the case of "no loss"), generates a significant linkage between the two problems described above, which is the issue analyzed in this work. In particular, this paper aims at showing that the presence of a common consequence in the comparison between two lotteries generates clear specific results which relate this comparison to the degree of agent risk aversion. Moreover, the application of these results show their implications for the choice between risky challenges and for the choice between self-protection and self-insurance.

The problems described above present situations where an agent either obtains a reward in the case of success in a challenge or suffers a loss in the case of a bad event, and both the reward and the loss are known with certainty. In many cases, however, successes generate rewards whose values are not exactly known, just as the consequences of losses may not be precisely known. In these cases, we have respectively random rewards in the choice between risky challenges and random losses in the choice between self-protection and self-insurance. Both these cases will be analyzed in the paper.

Lastly, a different kind of uncertainty may also be considered. When choosing between two challenges or between self-protection and self-insurance, an agent may face uncertainty on his background conditions, a further kind of uncertainty, which is independent of both the risk associated with the challenges and the risk of incurring financial loss. This kind of uncertainty, related to the presence of what is usually called in risk theory a "background risk", is also analyzed in the present work. 
The paper proceeds as follows. Section Two examines the comparison between lotteries with a common consequence. Section Three studies the application to the choice between risky challenges. Section Four analyzes the application to the choice between self-protection and self-insurance. Section Five concludes.

\section{Lotteries with a common consequence}

\subsection{Lotteries with a common lower consequence}

We define the concept of lotteries with a common lower consequence as follows:

Definition 1. The lotteries $A=\left[x_{0}, p ; x_{1}, 1-p\right]$ where $x_{0}<x_{1}$ and $B=\left[y_{0}, q ; y_{1}, 1-q\right]$ where $y_{0}<y_{1}$ have a common lower consequence if $x_{0}=y_{0}$.

Given Definition 1, our goal is to examine the choice between Lotteries A and B. Lotteries A and B have the same lower consequence while they have different upper consequences. Assume, without loss of generality, that $x_{1}<y_{1}$ and $p<q$, so that the lottery with the higher upper outcome also exhibits the lower probability that this outcome occurs.

We study in this work how preferences affect the choice between Lottery A and Lottery B. In order to implement this analysis we recall the Independence Axiom, which is on the basis of expected utility theory. A version of this Axiom useful for our paper is the following:

Axiom 1. Given the three lotteries $J, K$ and $L$, if an agent prefers (is indifferent between) Lottery J over (and) Lottery $K$, he also prefers (is indifferent between) the lottery $\alpha J+(1-\alpha) L$ over (and) the lottery $\alpha K+(1-\alpha) L$, where $\alpha$ is a probability.

Given this, considering the two agents $u$ and $v$ whose preferences are respectively described by functions $U($.$) and V($.$) we obtain:$

Proposition 1. Agent $v$ is more prone to choosing Lottery A than Agent $u$ if Agent $v$ is more risk averse than Agent $u$.

Proof. Consider Lotteries $A$ and $B$. Lottery $A$ is equivalent to the lottery $\left[x_{0} ; p ; A^{\prime}, 1-p\right]$ where $A^{\prime}=\left[x_{1}, 1\right]$. Lottery $B$ is equivalent to $\left[x_{0}, p ; x_{0}, q-p ; y_{1}, 1-q\right]$, which is, in turn, equivalent to the lottery $\left[x_{0}, p ; B^{\prime} ; 1-p\right]$ where $B^{\prime}=\left[x_{0}, t ; y_{1}, 1-t\right]$ and $t=\frac{q-p}{1-p}$. Axiom 1 now implies that the comparison between Lotteries $A$ and $B$ for agents $u$ and 
$v$ is equivalent to the comparison between Lotteries $A^{\prime}$ and $B^{\prime}$.

Moreover, Agent $u$ is indifferent between A' and B' if and only if $U\left(x_{1}\right)=t U\left(x_{0}\right)+[1-$ t] $U\left(y_{1}\right)$. Assume now that function $V($.$) is an increasing and concave transformation$ of function $U($.$) , i.e. that V()=.H(U()$.$) where H($.$) is increasing and concave. By$ the definition of concavity we thus have that $V\left(x_{1}\right)=H\left(U\left(x_{1}\right)\right)=H\left(t U\left(x_{0}\right)+[1-\right.$ $\left.t] U\left(y_{1}\right)\right)>t H\left(U\left(x_{0}\right)\right)+[1-t] H\left(U\left(y_{1}\right)\right)=t V\left(x_{0}\right)+[1-t] V\left(y_{1}\right)$. This implies that agent $v$ prefers Lottery $A$ over Lottery $B$, proving Statement a) in the proposition.

The above analysis was derived under the assumption that all the outcomes in Lotteries $\mathrm{A}$ and $\mathrm{B}$ are certainly known. A different case is when we have $A=\left[x_{0}, p ; \tilde{x}_{1}, 1-p\right]$ and $B=\left[y_{0}, q ; \tilde{y}_{1}, 1-q\right]$ and where $\tilde{x}_{1}$ and $\tilde{y}_{1}$ are random variables with $x_{0}<E\left[\tilde{x}_{1}\right]$, $y_{0}<E\left[\tilde{y}_{1}\right]$ and $x_{0}=y_{0}$. In this context, given the previous assumptions, we obtain:

Proposition 2. Assume that random variable $\tilde{y}_{1}$ is defined over the support $\left[k_{2}, k_{3}\right]$ and random variable $\tilde{x}_{1}$ is defined over the support $\left[k_{0}, k_{1}\right]$ where $k_{2}>k_{1}$ and $k_{0}>x_{0}$. Then Agent $v$ is more prone to choosing Lottery A than Agent u if Agent $v$ is more risk averse than Agent $u$.

Proof. The same steps as in the proof of Proposition 1 show that Agent $u$ is indifferent between Lotteries $\mathrm{A}$ and $\mathrm{B}$ if $E\left[U\left(\tilde{x}_{1}\right)\right]=t U\left(x_{0}\right)+[1-t] E\left[U\left(\tilde{y}_{1}\right)\right.$ which is equivalent to

$$
E\left[U\left(\tilde{y}_{1}\right)\right]-E\left[U\left(\tilde{x}_{1}\right)\right]=t\left[E\left[U\left(\tilde{y}_{1}\right)\right]-U\left(x_{0}\right)\right]
$$

As in the proof of Proposition 1, the statement is proved if, assuming that $V()=$. $H(U()$.$) where H($.$) is increasing and concave, we have E\left[H\left(U\left(\tilde{x}_{1}\right)\right)\right]>t H\left(U\left(x_{0}\right)\right)+$ $[1-t] E\left[H\left(U\left(\tilde{y}_{1}\right)\right)\right]$ which is equivalent to

$$
E\left[H\left(U\left(\tilde{y}_{1}\right)\right)\right]-E\left[H\left(U\left(\tilde{x}_{1}\right)\right)\right]<t\left[E\left[H\left(U\left(\tilde{y}_{1}\right)\right)\right]-H\left(U\left(x_{0}\right)\right)\right]
$$

By (1), (2) is equivalent to

$$
\frac{E\left[H\left(U\left(\tilde{y}_{1}\right)\right)\right]-E\left[H\left(U\left(\tilde{x}_{1}\right)\right)\right]}{E\left[U\left(\tilde{y}_{1}\right)\right]-E\left[U\left(\tilde{x}_{1}\right)\right]}<\frac{\left[E\left[H\left(U\left(\tilde{y}_{1}\right)\right)\right]-H\left(U\left(x_{0}\right)\right)\right]}{E\left[U\left(\tilde{y}_{1}\right)\right]-U\left(x_{0}\right)}
$$

Given our assumptions on $k_{2}, k_{1}, k_{0}$ and $x_{0}$ we have that $\tilde{y}_{1}$ dominates $\tilde{x}_{1}$ via first-order stochastic dominance and $\tilde{y}_{1}$ dominates $x_{0}$ via first-order stochastic dominance. Since $U($.$) is an increasing function, this implies that U\left(\tilde{y}_{1}\right)$ dominates $U\left(\tilde{x}_{1}\right)$ via first-order stochastic dominance and $U\left(\tilde{y}_{1}\right)$ dominates $U\left(x_{0}\right)$ via first-order stochastic dominance. 
This makes it possible to apply Theorem 4.1 by Di Crescenzo (1999) (see Lemma 1 in Appendix A) obtaining that (3) is equivalent to:

$$
\begin{gathered}
E\left[H^{\prime}(\tilde{z})\right]=E\left[H^{\prime}(\tilde{z})\right] \frac{E\left[U\left(\tilde{y}_{1}\right)\right]-E\left[U\left(\tilde{x}_{1}\right)\right]}{E\left[U\left(\tilde{y}_{1}\right)\right]-E\left[U\left(\tilde{x}_{1}\right)\right]}< \\
<\frac{E\left[U\left(\tilde{y}_{1}\right)\right]-U\left(x_{0}\right)}{E\left[U\left(\tilde{y}_{1}\right)\right]-U\left(x_{0}\right)} E\left[H^{\prime}(\tilde{s})\right]=E\left[H^{\prime}(\tilde{s})\right]
\end{gathered}
$$

where $\tilde{z}$ and $\tilde{s}$ are random variables. Now, since $H^{\prime \prime}()<0,. E\left[H^{\prime}(\tilde{z})\right]<E\left[H^{\prime}(\tilde{s})\right]$ if $\tilde{z}$ dominates $\tilde{s}$ via first order stochastic dominance. Appendix B proves that this is the case. This last step concludes the proof.

The last case we examine is the case where we add a symmetric mean-zero risk to each outcome of the lotteries defined in Definition $1 .^{3}$ We thus examine in this case the two lotteries $A=\left[x_{0}+\tilde{\epsilon}, p ; x_{1}+\tilde{\epsilon}, 1-p\right]$ and $B=\left[y_{0}+\tilde{\epsilon}, q ; y_{1}+\tilde{\epsilon}, 1-q\right]$ where $x_{0}<x_{1}$, $y_{0}<y_{1}, x_{0}=y_{0}$ and $\tilde{\epsilon}$ is a symmetric mean-zero random variable.

In order to study this case we need to introduce two concepts. Brockett and Golden (1987) and Caballé and Pomanski (1996) define the concept of mixed risk averse agent as follows:

Definition 2. An agent is mixed risk averse when $(-1)^{i+1} \frac{d^{i} U(z)}{d z^{i}}>0$ for $i=1, \ldots \infty$.

Mixed risk aversion is thus the case where all the odd derivatives of agent's utility function are positive and all the even derivatives are negative. This definition implies that each mixed risk averse agent is risk averse but not vice versa. ${ }^{4}$

In a recent paper Crainich et al. (2013) also introduced the concept of mixed risk loving, which is defined as follows:

Definition 3. An agent is a mixed risk lover when $\frac{d^{i} U(z)}{d z^{i}}>0$ for $i=1, \ldots \infty$

Mixed risk loving is thus the case where all the derivatives of the utility function are positive. This definition implies that a mixed risk lover is a risk lover but not vice versa. $^{5}$

\footnotetext{
${ }^{3}$ We have, for instance, a symmetric distribution when the risk has normal or uniform distribution, but clearly this also occurs for many other distributions.

${ }^{4}$ Eeckhoudt and Schlesinger (2006) show that mixed risk aversion can be seen as the wish of risk apportionment in the sense that an agent who exhibits these preferences desires to implement different kinds of harm disaggregation. Mixed risk aversion has also proved to be relevant in many economic applications (e.g. Eeckhoudt and Schlesinger, 2008; Wang et al., 2009; Crainich et al., 2016). Lastly, on the characterization of mixed risk aversion, see also Menegatti (2015).

${ }^{5}$ Crainich et al (2013) show that a mixed risk lover makes precautionary saving while Crainich et al. (2016) show the role of mixed risk loving in the choice of optimal prevention.
} 
We now compare the choice between Lotteries A and B of two agents: Agent $u$, whose preferences are described by the utility function $U($.$) and the risk neutral agent.$ We get that:

Proposition 3. a) If Agent $u$ is mixed risk averse then he is more prone to choosing Lottery $A$ than the risk neutral agent.

b) If Agent $u$ is a mixed risk lover then he is more prone to choosing Lottery $B$ than the risk neutral agent.

Proof. The same steps as in the proof of Proposition 1 imply that the comparison between Lotteries $A$ and $B$ is equivalent to the comparison between $A^{\prime}=\left[x_{1}+\tilde{\epsilon}, 1\right]$ and $B^{\prime}=\left[x_{0}+\tilde{\epsilon}, t ; y_{1}+\tilde{\epsilon}, 1-t\right]$.

We assume that the risk neutral agent is indifferent between the two lotteries, which implies $x_{1}=t x_{0}+(1-t) y_{0}$, implying in turn

$$
y_{1}-x_{1}=t\left(y_{1}-x_{0}\right)
$$

Now Agent $u$ prefers Lottery $A$ over Lottery $B$ if $E\left[U\left(x_{1}+\tilde{\epsilon}\right)\right]>t E\left[U\left(x_{0}+\tilde{\epsilon}\right)\right]+[1-$ $t] E\left[U\left(y_{1}+\tilde{\epsilon}\right)\right]$, which is equivalent to

$$
E\left[U\left(y_{1}+\tilde{\epsilon}\right)\right]-E\left[U\left(x_{1}+\tilde{\epsilon}\right)\right]<t\left[E\left[U\left(y_{1}+\tilde{\epsilon}\right)\right]-E\left[U\left(x_{0}+\tilde{\epsilon}\right)\right]\right.
$$

and prefers Lottery $B$ over Lottery $A$ if the reversed inequality holds.

In order to study (6), consider now that, given the generic random variable $\mathrm{X}$, we have, by using a Taylor expansion:

$$
E[f(X)]=\sum_{n=0}^{+\infty} \frac{f^{(n)}(E[X]) E\left[(X-E[X])^{n}\right]}{n !}
$$

where $f^{(0)}=f$ and $f^{(n)}$ is the nth-order derivative of function $f$. Note that Equation (7) is not an approximation (because the Taylor expansion in it is not truncated), so the exact equality holds and the results derived below hold for risks of any size. Applying (7) to (6) we get

$$
\sum_{n=0}^{+\infty}\left[U^{(n)}\left(y_{1}\right)-U^{(n)}\left(x_{1}\right)\right] E\left[\tilde{\epsilon}^{n}\right]<\sum_{n=0}^{+\infty}\left[U^{(n)}\left(y_{1}\right)-U^{(n)}\left(x_{0}\right)\right] E\left[\tilde{\epsilon}^{n}\right]
$$

Since the distribution of $\tilde{\epsilon}$ is symmetric, $E\left[\tilde{\epsilon}^{j}\right]=0$ for every odd $j$ and $E\left[\tilde{\epsilon}^{j}\right]>0$ for every even $j$. This implies that (8) is equivalent to

$$
\sum_{n=0}^{+\infty}\left[U^{(2 n)}\left(y_{1}\right)-U^{(2 n)}\left(x_{1}\right)\right]<\sum_{n=0}^{+\infty}\left[U^{(2 n)}\left(y_{1}\right)-U^{(2 n)}\left(x_{0}\right)\right]
$$


By applying the mean value Theorem we obtain that (9) is equivalent to

$$
\left(y_{1}-x_{1}\right) \sum_{n=0}^{+\infty} U^{(2 n+1)}(z)<t\left(y_{1}-x_{0}\right) \sum_{n=0}^{+\infty} U^{(2 n+1)}(s)
$$

where $z>s$ and which, by (5), is in turn equivalent to

$$
\sum_{n=0}^{+\infty} U^{(2 n+1)}(z)<\sum_{n=0}^{+\infty} U^{(2 n+1)}(s)
$$

Now in case of mixed risk aversion, we have that $U^{(2 n+1)}($.$) is decreasing for each n$. This implies that Inequality (11) is satisfied, proving Statement a).

In case of mixed risk loving, we have instead that $U^{(2 n+1)}($.$) is increasing for each n$. This implies that the opposite of Inequality (11) is satisfied, proving Statement b).

\subsection{Lotteries with a common upper consequence}

We define the concept of lotteries with a common upper consequence as follows:

Definition 4. The lotteries $A=\left[x_{0}, p ; x_{1}, 1-p\right]$, where $x_{1}>x_{0}$, and $B=\left[y_{0}, q, y_{1}, 1-\right.$ $q$ ], where $y_{1}>y_{0}$, have a common upper consequence if $x_{1}=y_{1}$.

Lotteries A and B have the same upper consequence, while they have different lower consequences. Similarly to what we did in Section 2.1, we assume, without loss of generality, that $x_{0}>y_{0}$ and $p>q$.

Considering now the two agents $u$ and $v$ whose preferences are respectively described by functions $U($.$) and V($.$) , we obtain:$

Proposition 4. Agent $v$ is more prone to choosing Lottery A than Agent u if Agent $v$ is more risk averse than Agent $u$.

Proof. Consider Lotteries $A$ and $B$. Lottery $A$ is equivalent to the lottery $\left[A^{\prime}, p ; x_{1}, 1-p\right]$ where $A^{\prime}=\left[x_{0}, 1\right]$. Lottery $B$ is equivalent to $\left[y_{0}, q ; x_{1}, 1-p ; x_{1}, p-q\right]$, which is, in turn, equivalent to the lottery $\left[B^{\prime}, p ; x_{1}, 1-p\right]$ where $B^{\prime}=\left[y_{0}, t ; x_{1}, 1-t\right]$ and $t=\frac{q}{p}$. Axiom 1 now implies that the comparison between Lotteries $A$ and $B$ for agents $u$ and $v$ is equivalent to the comparison between Lotteries $A^{\prime}$ and $B^{\prime}$. From this step the proof proceeds similarly to the proof of Proposition 1. 
The above analysis was derived under the assumption that all the outcomes in Lotteries A and B are certainly known. A different case is obtained when we have $A=$ $\left[\tilde{x_{0}}, p ; x_{1}, 1-p\right]$ and $B=\left[\tilde{y}_{0}, q ; y_{1}, 1-q\right]$ and where $\tilde{x}_{0}$ and $\tilde{y}_{0}$ are random variables with $x_{1}>E\left[\tilde{x}_{0}\right], y_{1}>E\left[\tilde{y}_{0}\right]$ and $x_{1}=y_{1}$. In this context, given the previous assumptions, we obtain:

Proposition 5. Assume that the random variable $\tilde{x_{0}}$ is defined over the support $\left[k_{2}, k_{3}\right]$ and the random variable $\tilde{y}_{0}$ is defined over the support $\left[k_{0}, k_{1}\right]$ where $x_{1}>k_{3}$ and $k_{2}>k_{1}$. Then Agent $v$ is more prone to choosing Lottery $A$ than Agent $u$ if and only if Agent $v$ is more risk averse than Agent $u$.

Proof. Similar to the proof of Proposition 2

The last case we examine is the case where we add a symmetric mean-zero risk to each outcome of the lotteries defined in Definition 1. We thus examine in this case the two lotteries $A=\left[x_{0}+\tilde{\epsilon}, p ; x_{1}+\tilde{\epsilon}, 1-p\right]$ and $B=\left[y_{0}+\tilde{\epsilon}, q ; y_{1}+\tilde{\epsilon}, 1-q\right]$ where $x_{0}<x_{1}$, $y_{0}<y_{1}, x_{1}=y_{1}$ and $\tilde{\epsilon}$ is a symmetric mean-zero random variable.

We compare the choice between Lotteries $\mathrm{A}$ and $\mathrm{B}$ of Agent $u$, whose preferences are described by the utility function $U($.$) , and the risk neutral agent. We obtain that:$

Proposition 6. a) If Agent $u$ is mixed risk averse then he is more prone to choosing Lottery $A$ than the risk neutral agent.

b) If Agent $u$ is a mixed risk lover then he is more prone to choosing Lottery $B$ than the risk neutral agent.

Proof. Similar to the proof of Proposition 3.

\section{The choice between risky challenges}

The comparison between lotteries with a common lower consequence can be used to study the choice between risky challenges. As shown in the introduction, this kind of challenge describes many possible situations, such as the choice between different investment projects, between different application for grants or funding or between different sport competitions.

We assume that an agent can undertake two possible actions, called hereafter "challenges". The two challenges have the same cost $c \geq 0$ and each of them can generate two possible outcomes: either success or failure. In the case of failure, both challenges 
produce the same outcome: agent status does not change. So, given the present level of wealth $\bar{W}_{0}$, in the case of failure, agent level of wealth is $W_{0}=\bar{W}_{0}-c>0$. On the other hand, in the case of success, the two challenges have different rewards. If the agent succeeds in the first challenge, his wealth increases to the level $W_{1}>0$ (where $W_{1}$ is net wealth, already including the payment of the cost $\left.c\right) .{ }^{6}$ Success in the second challenge instead implies that wealth becomes equal to $W_{1}-m>0$.

We assume that, in both challenges, wealth is larger in the case of success than in the case of failure. We also assume that the wealth level in the case of success is larger in the first challenge than in the second. These assumptions obviously imply $W_{1}>W_{1}-m>W_{0}$, which implies in turn $m>0$. We also assume that the probability of failure is equal to $p$ in the first challenge and to $p-\Delta$ in the second challenge, where $p>\Delta>0$. This clearly implies that the probabilities of success in the two challenges are respectively $1-p$ and $1-p+\Delta$. Moreover, the assumptions on $p$ and $\Delta$ together with those on $W_{0}, W_{1}$ and $m$ imply that the first challenge exhibits a higher level of wealth in the case of success but a lower probability of succeeding. We call this challenge the "hard challenge". In parallel, the second challenge exhibits a lower level of wealth in the case of success but a higher probability of succeeding. We call this challenge the "soft challenge".

We now assume that the agent cannot take up both challenges and must choose between them. This idea can be justified in various ways. First, there may be some kind of time constraint, implying that the agent cannot contemporaneously compete in both challenges. Second, the cost of taking up both challenges may be too high for the agent. Since taking up the two challenges together implies paying cost $c$ twice, this may occur, for instance, when $W_{1}-m>W_{0}>W_{1}-m-c$. Lastly, taking up both challenges together is sometimes not permitted by law or by regulations.

Given the framework described, the two challenges alternatively faced by the agent can be analyzed by studying the lotteries $\left[W_{0}, p ; W_{1}, 1-p\right]$ for the hard challenge and $\left[p-\Delta, W_{0} ; 1-p+\Delta, W_{1}-m\right]$ for the soft challenge, which are depicted in Figure 1. We assume that the agent chooses which challenge to take up on the basis of his preferences.

As in the previous section, we now compare the optimal choices of agents $u$ and $v$ whose preferences are described by functions $U($.$) and V($.$) respectively. This compar-$ ison generates the following conclusion.

\footnotetext{
${ }^{6}$ As for $W_{0}$, we may define $W_{1}=\bar{W}_{1}-c$, where $\bar{W}_{1}$ is gross wealth (excluding the cost $c$ ).
} 
Figure 1: The choice between the risky challenges

Hard Challenge

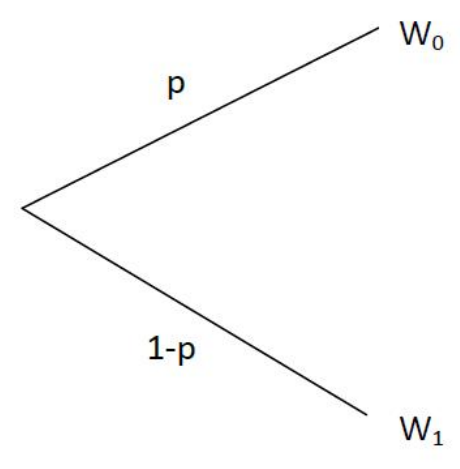

Soft Challenge

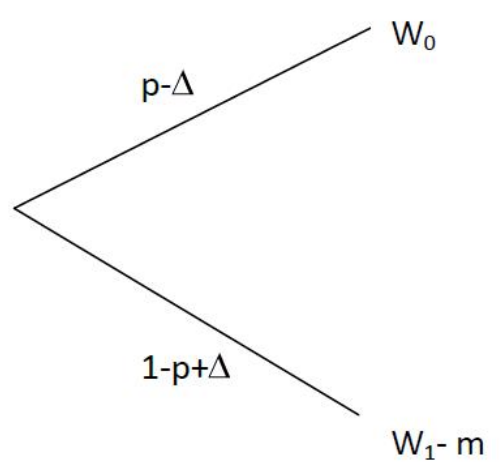

Result 1. Agent $v$ is more prone to choosing the soft challenge than Agent u if Agent $v$ is more risk averse than Agent $u$.

Proof. Directly derived from Proposition 1

The conclusions highlighted in Result 1 can be graphically illustrated in a simplified example. Assume, for simplicity, that the soft and the hard challenge yield the same expected wealth and that Agent $u$ is risk neutral. These assumptions imply that Agent $u$ is indifferente between the two challenges. We now draw, in this context, the cumulative distribution functions associated with the hard and soft challenges (see Figure 2).

As the figure clearly shows, the cumulative distribution function associated with the soft challenge starts lower than that associated with the hard challenge (the lowest outcome $W_{0}$ is obtained with the probabilities $p$ and $p-\Delta$ under the hard and soft challenges respectively) and finishes above it ( $W_{1}$, the best outcome obtained under the hard challenge is higher than $W_{1}-m$ which is at best obtained under the soft one). As a result, the cumulative distribution function associated with the soft challenge crosses that associated with the hard challenge once from below. Moreover, since the two challenges have the same expected value, the areas below the two cumulative distribution functions are equal. All this implies that, in our illustration, the hard challenge is an increase in risk á la Rotshcild and Stiglitz (1970) compared to the soft challenge. This implies in turn that if Agent $v$ is risk averse then he prefers the soft challenge over the hard challenge. 
Figure 2: The cumulative distribution functions of the risky challenges

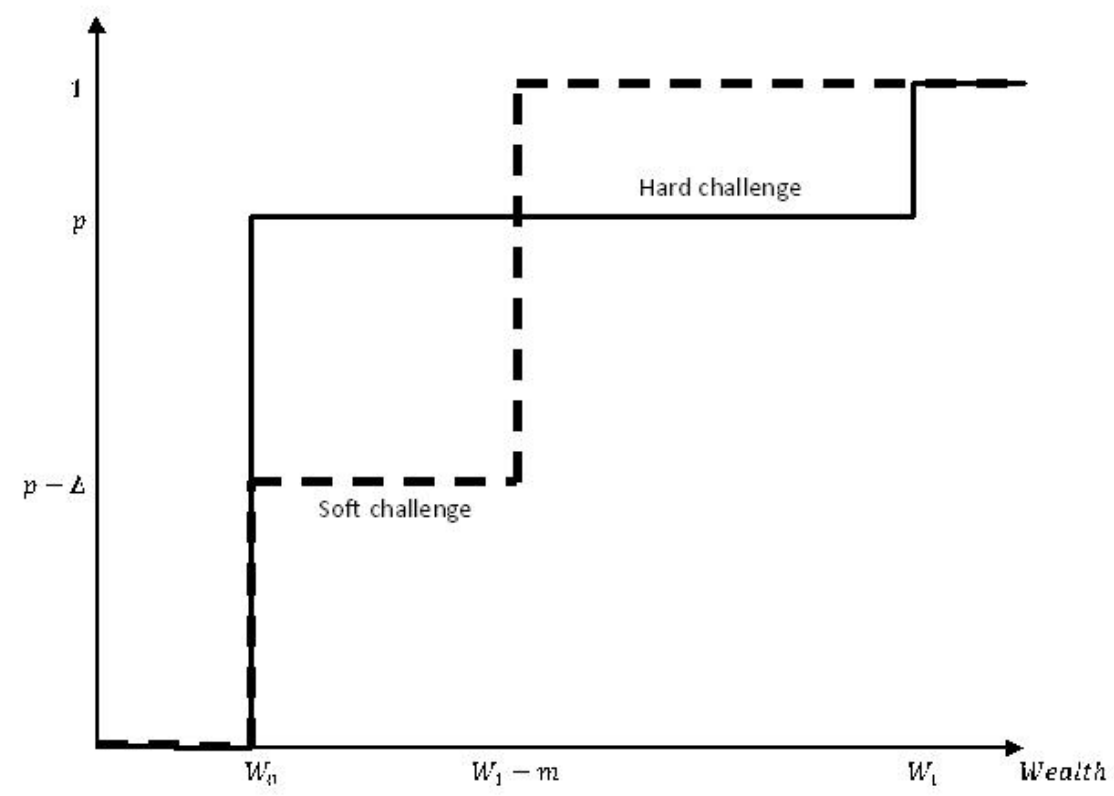

\subsection{Random rewards}

We examined above a model where an agent chooses between two different challenges where the rewards in the case of success are known with certainty. In many cases, however, we know if the reward in the case of success in a challenge is high or low, but we do not know its exact value. This kind of uncertainty may involve both challenges compared by the agents. In this case, the lottery describing the hard challenge is $\left[W_{0}, p ; \tilde{W}_{1}, 1-p,\right]$ while the lottery describing the soft challenge is $\left[W_{0}, p-\Delta ; \tilde{W}_{1}-\right.$ $\tilde{m}, 1-p+\Delta]$, where $\tilde{W}_{1}$ and $\tilde{m}$ are two random variables.

We compare, as in previous sections, the choice of agents $u$ and $v$, obtaining the following results:

Result 2. Assume $\tilde{W}_{1} \in\left[\alpha_{0}, \alpha_{1}\right]$ and $\tilde{m} \in\left[\alpha_{2}, \alpha_{3}\right]$ where $\alpha_{0}-\alpha_{3}>W_{0}$ and $\alpha_{2}>0$. Then Agent $v$ is more prone to choosing the soft challenge than Agent $u$ if Agent $v$ is more risk averse than Agent $u$.

Proof. The proof is directly derived from Proposition 2.

In the absence of uncertainty on the rewards, we showed that the risk averse agent is more prone than the risk neutral agent to choosing the soft challenge. If we introduce random rewards, we add further sources of uncertainty to the problem, which 
the risk averse agent does not like. We may thus expect that this would make the comparison between the two challenges ambiguous for the risk averse agent. Result 2 shows, however, that the same clear conclusion obtained in the case of given rewards holds, under some plausible assumptions on the random variables describing random rewards. These assumptions simply require that the variability of the random reward is constrained within an interval which ensures that the level of wealth obtained in case of success in the soft challenge is certainly higher than the level of wealth in case of failure, and is certainly lower than the level of wealth obtained in case of success in the hard challenge. If these conditions hold, additional uncertainty is limited enough to ensure that the more risk averse agent is still more prone to choosing the soft challenge.

\subsection{Background risks}

In Result 2, we examined the effect of the introduction of random rewards in the case of success in the two alternative challenges faced by the agent. A different kind of uncertainty, which an agent may face when choosing between the two challenges, refers to his background conditions. This kind of uncertainty is independent of the challenge he chooses to face and independent of success or failure in it, and can be studied by introducing a background risk in the setting described in Figure 1.

In this case the lottery describing the hard challenge becomes $\left[W_{0}+\tilde{\epsilon}, p ; W_{1} \tilde{\epsilon}, 1-p\right]$ while the lottery describing the soft challenge becomes $\left[W_{0} \tilde{\epsilon}, p-\Delta ; W_{1}-m \tilde{\epsilon}, 1-p+\Delta\right]$, where $\tilde{\epsilon}$ is a symmetric zero-mean random variable.

Under these assumptions we compare the optimal choice of Agent $u$, whose preferences are described by the utility function $U($.$) , with the optimal choice of the risk$ neutral agent. We obtain:

Result 3. a) If Agent $u$ is mixed risk averse then he is more prone to choosing the soft challenge than the risk neutral agent.

b) If Agent $u$ is a mixed risk lover then he is more prone to choosing the hard challenge than the risk neutral agent.

Proof. Directly derived from Proposition 3

Result 3 shows that, in the presence of a background risk, we obtain a more specific conclusion than in the analysis in the previous subsections. In this case, in fact, our findings require the stronger condition that the agent is either mixed risk averse or a 
mixed risk lover, which implies the introduction of constraints on the sign of all the subsequent derivatives of the utility function. The results obtained show that mixed risk aversion pushes the agent to choose the soft challenge and mixed risk loving pushes the agent to choose the hard challenge.

\section{The choice between self-protection and self-insurance}

The choice between lotteries with an upper common consequence analyzed in Section 2.2 can be used to study the choice between self-protection and self-insurance in a context where an agent faces the risk of incurring a loss. Many examples of this situation are provided in Section 1.

We consider an agent who has an initial wealth equal to $W_{0}$ and faces the risk of incurring the loss $L$ with probability $p$. Without any action, the agent will thus have a wealth equal to $W_{0}-L$ with probability $p$ and equal to $W_{0}$ with probability $1-p$.

The agent can implement two alternative actions. The first action is a self-protection activity. Self-protection is defined in risk theory as an activity which reduces the probability of incurring loss $L$ without affecting its size. If the agent implements selfprotection, the probability of incurring the loss becomes $p-\Delta$ with $p>\Delta>0$, instead of $p$. The probability of no loss thus becomes $1-p+\Delta$.

The second activity that the agent can implement is a self-insurance activity. Risk theory defines self-insurance as an activity which reduces the size of the possible loss $L$ without affecting the probability that it occurs. If the agent implements self-insurance he will incur a loss $L-m$ (where $m>0$ ) with probability $p$, while his wealth will be unchanged with probability $1-p$.

We now assume that the agent has a budget $c \geq 0$ which is fully allocated to one of the two activities and we define $W=W_{0}-c$. We assume that the expected value of wealth both in the case of self-protection activity and in the case of self-insurance activity is higher than in the case of no action implemented. We also assume that the budget is not sufficient to implement both activities together. This implies that the agent has to choose between the self-protection activity and the self-insurance activity. The same situation can be obtained under different assumptions, as, for instance, the presence of a budget constraint, which limits the tolerable costs, or in the presence of time constraints. The choice the agent has to make is also represented in Figure 3.

As in previous sections, we now compare the two agents $u$ and $v$, whose preferences 
Figure 3: The choice between self-protection and self-insurance
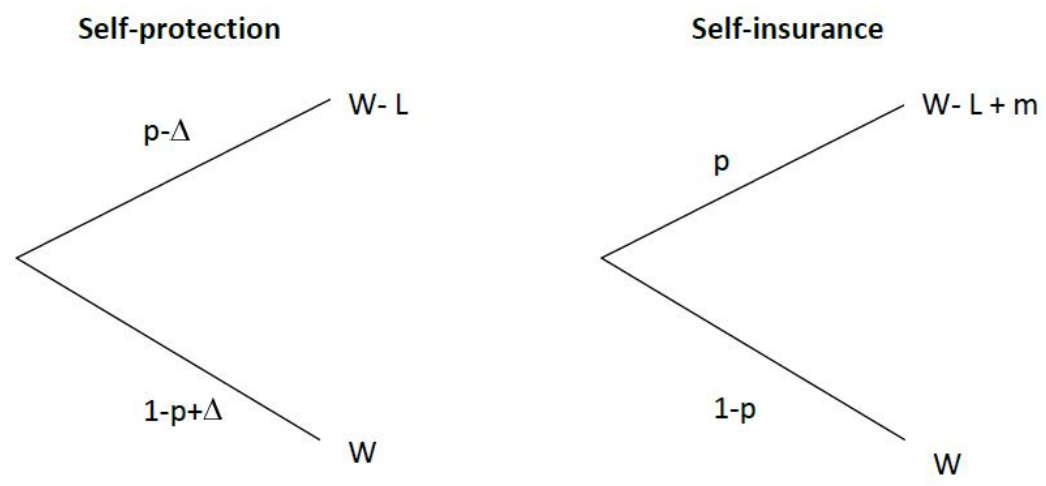

are described by utility functions $U($.$) and V($.$) respectively. We obtain the following$ results:

Result 4. Agent $v$ is more prone to choosing self-insurance than Agent u if Agent $v$ is more risk averse than Agent $u$.

Proof. The proof directly drives from Proposition 4.

The cumulative distributions of self-protection and self-insurance are represented in Figure 4. Both activities are supposed to yield the same expected wealth so that a riskneutral agent is indifferent between the two prevention options. Since wealth in case of loss is lower under self-protection than self-insurance, the cumulative distribution function of the former rises before (at wealth $W-L$ ) that of the latter (that rises at wealth level $W-L+m$ ). Besides, both distribution finishes at the same wealth level (the common consequence $W$ ) and the areas below the cumulative distributions are equal since both activities yield the same expected wealth. Consequently, the cumulative distribution function of self-insurance crosses that of self-protection once from below, which means that self-insurance constitutes an increase in risk á la Rotschild and Stiglitz (1970) compared to self-protection. As a result, risk averse agents prefer self-insurance over self-protection in the illustration provided.

\subsection{Random losses}

The analysis above studies the case where an agent faces the risk of incurring a loss whose size is given. In some cases, however, we know that we will incur a loss but 
Figure 4: The cumulative distribution functions of self-protection and self-insurance

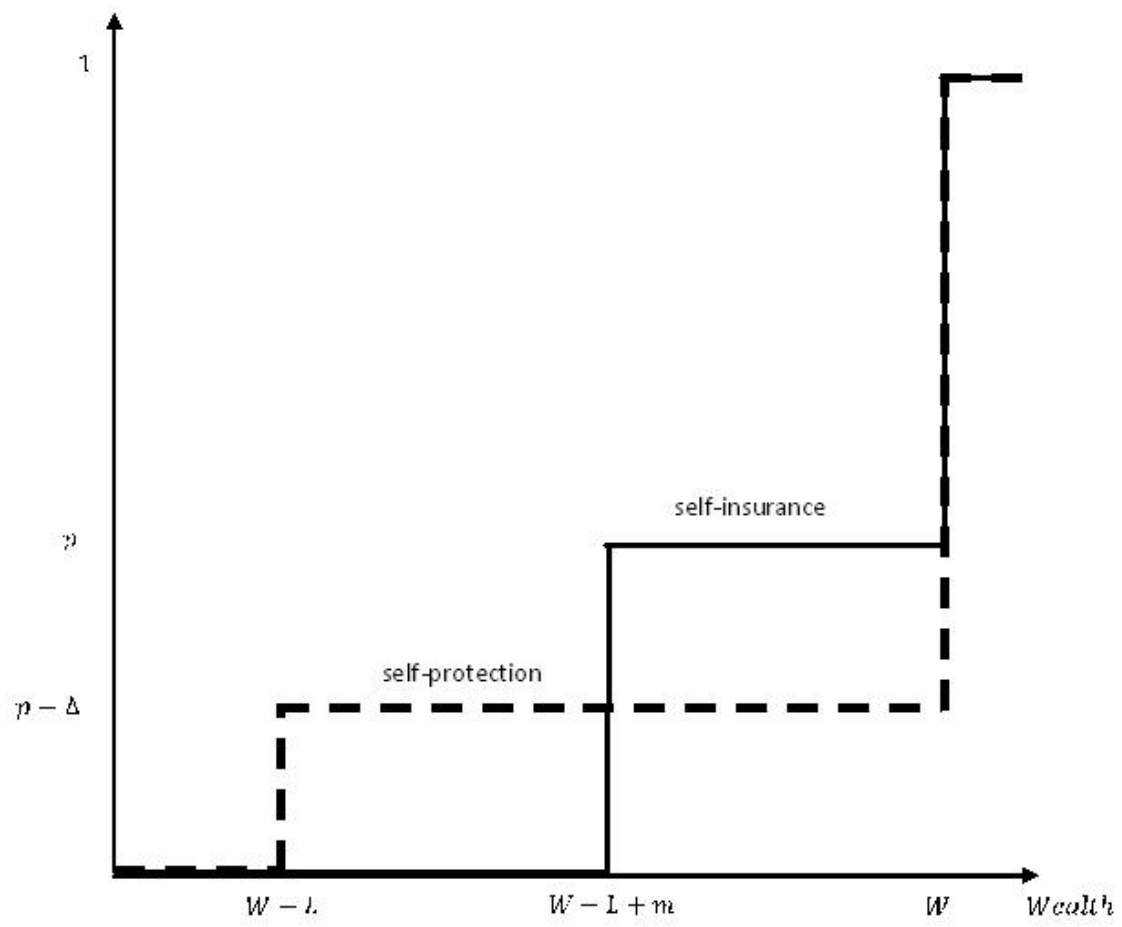

we do not know its exact value. In this case, loss can be represented by the random variable $\tilde{L}$. We also assume that, when the agent chooses to implement a self-insurance activity the loss will become $\tilde{L}-\tilde{m}$. This hypothesis implicitly assumes that the effect of self-insurance is not known either with certainty.

We now compare the two agents $u$ and $v$, whose preferences are described by utility functions $U($.$) and V($.$) respectively. We obtain the following results:$

Result 5. Assume that $\tilde{L} \in\left[\beta_{0}, \beta_{1}\right]$ and $\tilde{m} \in\left[\beta_{2}, \beta_{3}\right]$ where $\beta_{0}>\beta_{3}$ and $\beta_{2}>0$. Then Agent $v$ is more prone to choosing self-insurance than Agent $u$ if Agent $v$ is more risk averse than Agent $u$.

Proof. The proof directly derives from Proposition 5.

The introduction of uncertainty on the level of possible loss $L$ and on the mitigating effect of self-insurance, represented by $m$, implies that we get clear-cut conclusions only when this uncertainty is limited. In particular, Result 5 shows that, in this framework, the more risk averse agent is more prone to choosing self-insurance when the variability 
of $\tilde{L}$ and $\tilde{m}$ is sufficiently limited to ensure that, even after the mitigating effect of selfinsurance, the random loss cannot be negative (i.e. it cannot become a gain because of self-insurance) and that the level of the loss with self-insurance is certainly lower than the level in the absence of self-insurance (i.e. that self-insurance always mitigates the loss).

\subsection{Background risks}

The issue of dealing with the risk of incurring loss may also be studied in a context where the background conditions of the decision maker are not certainly known. This means considering the risk of incurring the loss $L$ together with a background risk, i.e. introducing a background risk into the setting described in Figure 3.

In this case, the lottery describing self-protection becomes $[W-L+\tilde{\epsilon}, p-\Delta ; W+\tilde{\epsilon}, 1-$

$p+\Delta]$ while the lottery describing self-insurance becomes $[W-L+m+\tilde{\epsilon}, p ; W+\epsilon, 1-p]$, where $+\tilde{\epsilon}$ is a symmetric zero-mean random variable.

Under these assumptions, we compare the optimal choice of Agent $u$, whose preferences are described by the utility function $U($.$) , with the optimal choice of the risk$ neutral agent. We obtain:

Result 6. a) If Agent $u$ is mixed risk averse then he is more prone to choosing selfinsurance than the risk neutral agent.

b) If Agent $u$ is a mixed risk lover then he is more prone to choosing self-protection than the risk neutral agent.

Proof. Directly derived from Proposition 6

Result 6 show that, in the presence of a background risk, we obtain a clear-cut conclusion on the optimal choice between self-protection and self-insurance only when the stronger assumptions of either mixed risk aversion or mixed risk loving are introduced. The findings show that mixed risk aversion pushes the agent to choose self-insurance while mixed risk loving pushes the agent to choose self-protection.

\section{Conclusions}

This work studies the choice between two binary lotteries which exhibit a common consequence (either in the bad state of nature or in the good state of nature). The analysis of this choice is implemented in cases where all the possible outcomes of the 
two lotteries are non-random, where the different outcomes are random, and lastly in the presence of an additional background risk. In all three cases, we derive results on how aspects of attitude toward risk affect the optimal choice.

These results are then applied to the choice between two risky challenges characterized by different rewards in the case of success and by different probabilities of succeeding. In this problem we obtain the following conclusions. First, in the case of non-random rewards, more risk aversion pushes an agent to choose the challenge with the lower reward in the case of success but the higher probability of succeeding. The same conclusion holds when the rewards are random but only if the random variables describing rewards satisfy some constraints. Lastly, when a background risk is introduced, mixed risk aversion pushes the agent to choose the challenge with the lower reward in the case of success but the higher probability of succeeding, while mixed risk loving pushes the agent to choose the challenge with the higher reward in the case of success but the lower probability of succeeding.

A second application involves the comparison between self-protection and self-insurance activities for an agent facing the risk of incurring a loss. The conclusions obtained are the following. In the case where the levels of the possible loss and of the mitigating effect of self-insurance are known with certainty, more risk aversion pushes an agent to choose the self-insurance activity. A similar result holds when these levels are random, but only under certain constraints on the random variables describing them. Lastly, when a background risk is introduced, mixed risk aversion pushes the agent to choose the self-insurance activity, and mixed risk loving pushes the agent to choose the selfprotection activity.

The issue examined in this work is, to our knowledge, new to the literature. This implies that future research could study generalizations of the framework analyzed in various directions, such as non-binary lotteries, multi-period problems and sequential choice between the lotteries.

\section{Appendix A}

Lemma 1. Given the two non-negative random variables $X$ and $Y$ satisfying $Y_{F S D} \geq$ $X$ and $E[X]<E[Y]<+\infty$, let $Z$ be the random variable with probability density function

$$
f_{Z}(t)=\frac{\bar{F}_{Y}(t)-\bar{F}_{X}(t)}{E[Y]-E[X]}
$$


with $t \geq 0$ and where $\bar{F}_{X}$ and $\bar{F}_{Y}$ are the survival functions of $X$ and $Y$. If $g($.$) is a$ measurable and differentiable function such that $E[g(X)]$ and $E[g(Y)]$ are finite then $E\left[g^{\prime}(Z)\right]$ is finite and

$$
E[g(Y)]-E[g(X)]=E\left[g^{\prime}(Z)\right][E[Y]-E[X]]
$$

Proof. See Di Crescenzo (1999), Proposition 3.1 and Theorem 4.1 .

\section{Appendix B}

We prove here that $\tilde{z}$ dominates $\tilde{s}$ via first order stochastic dominance. Given the functions $F_{\tilde{z}}($.$) and F_{\tilde{s}}($.$) which are the cumulative distribution functions of \tilde{s}$ and $\tilde{z}, \tilde{z}$ dominates $\tilde{s}$ via first order stochastic dominance if $G(j)=F_{\tilde{s}}(j)-F_{\tilde{z}}(j) \geq 0$ for every $j$ with a strict inequality for some $j$. Also note that $\frac{d G}{d j}=f_{\tilde{s}}(j)-f_{\tilde{z}}(j)$ where $f_{\tilde{s}}(j)$ and $f_{\tilde{z}}(j)$ are the density functions of $\tilde{s}$ and $\tilde{z}$ respectively. By applying Proposition 3.1 in Di Crescenzo (1999) (see again Appendix A), we obtain:

$$
f_{\tilde{z}}(j)= \begin{cases}0 & k_{0}>j \\ \frac{1-\bar{F}_{U\left(\tilde{x}_{1}\right)}(j)}{E\left[U\left(\tilde{y}_{1}\right)\right]-E\left[U\left(\tilde{x}_{1}\right]\right)} & k_{1}>j \geq k_{0} \\ \frac{1}{E\left[U\left(\tilde{y}_{1}\right)\right]-E\left[U\left(\tilde{x}_{1}\right]\right)} & k_{2}>j \geq k_{1} \\ \frac{\bar{F}_{U\left(\tilde{y}_{1}\right)}(j)}{E\left[U\left(\tilde{y}_{1}\right)\right]-E\left[U\left(\tilde{x}_{1}\right]\right)} & k_{3}>j \geq k_{2} \\ 0 & j \geq k_{3}\end{cases}
$$

where $\bar{F}_{U\left(\tilde{x}_{1}\right)}(j)$ and $\bar{F}_{U\left(\tilde{y}_{1}\right)}(j)$ denote the survival function of the random variables $U\left(\tilde{x}_{1}\right)$ and $U\left(\tilde{y}_{1}\right)$ respectively, and

$$
f_{\tilde{s}}(j)= \begin{cases}0 & x_{0}>j \\ \frac{1}{E\left[U\left(\tilde{y}_{1}\right)\right]-U\left(x_{0}\right)} & k_{2}>j \geq x_{0} \\ \frac{\bar{F}_{U\left(\tilde{y}_{1}\right)}(j)}{E\left[U\left(\tilde{y}_{1}\right)\right]-U\left(x_{0}\right)} & k_{3}>j \geq k_{2} \\ 0 & j \geq k_{3}\end{cases}
$$


This implies that:

$$
f_{\tilde{s}}(j)-f_{\tilde{z}}(j)= \begin{cases}0 & x_{0}>j \\ \frac{1}{E\left[U\left(\tilde{y}_{1}\right)\right]-U\left(x_{0}\right)} & k_{0}>j \geq x_{0} \\ \frac{1}{E\left[U\left(\tilde{y}_{1}\right)\right]-U\left(x_{0}\right)}-\frac{1-\bar{F}_{U\left(\tilde{x}_{1}\right)}(j)}{E\left[U\left(\tilde{y}_{1}\right)\right]-E\left[U\left(\tilde{x}_{1}\right]\right)} & k_{1}>j \geq k_{0} \\ \frac{1}{E\left[U\left(\tilde{y}_{1}\right)\right]-U\left(x_{0}\right)}-\frac{1}{E\left[U\left(\tilde{y}_{1}\right)\right]-E\left[U\left(\tilde{x}_{1}\right]\right)} & k_{2}>j \geq k_{1} \\ \frac{\bar{F}_{U\left(\tilde{y}_{1}\right)}(j)}{E\left[U\left(\tilde{y}_{1}\right)\right]-U\left(x_{0}\right)}-\frac{\bar{F}_{U\left(\tilde{y}_{1}\right)}(j)}{E\left[U\left(\tilde{y}_{1}\right)\right]-E\left[U\left(\tilde{x}_{1}\right]\right)} & k_{3}>j \geq k_{2} \\ 0 & j \geq k_{3}\end{cases}
$$

It is clear that $f_{\tilde{s}}(j)<f_{\tilde{z}}(j)$ for $j \geq k_{1}$ and that $f_{\tilde{s}}(j)>f_{\tilde{z}}(j)$ for $j<x_{0}$. Moreover, since $\bar{F}_{U\left(\tilde{x}_{1}\right)}\left(k_{1}\right)=0$ and $\bar{F}_{U\left(\tilde{x}_{1}\right)}\left(k_{0}\right)=1$ and $\bar{F}_{U\left(\tilde{x}_{1}\right)}($.$) is a decreasing function in the$ interval $\left[k_{0}, k_{1}\right]$ there exist a value $j_{0} \in\left[k_{0}, k_{1}\right]$ such that $f_{\tilde{s}}(j)<f_{\tilde{z}}(j)$ for $j>j_{0}$ and that $f_{\tilde{s}}(j)>f_{\tilde{z}}(j)$ for $j<j_{0}$. All this implies that $G(j)=0$ for $j \geq k_{3}, G(j)=0$ for $j<x_{0}, G(j)$ increasing for $j<j_{0}$ and $G(j)$ decreasing for $j>j_{0}$. This implies, in turn, that $G(j) \geq 0 \forall j \in\left[x_{0}, k_{3}\right]$ with a strict inequality for some $j$, which, as shown above, means that $\tilde{z}$ dominates $\tilde{s}$ via first order stochastic dominance.

\section{References}

[1] Briys, E., Schlesinger, H. (1990) Risk aversion and the propensities for self-insurance and self-protection. Southern Economic Journal 57, 458-467.

[2] Brochett, P.L., Golden, L.L. (1985) A class of utility functions containing all the common utility functions. Management Science 33, 955-964.

[3] Caballé, J., Pomanski, A. (1996) Mixed risk aversion. Journal of Economic Theory $71,485-513$.

[4] Crainich, D., Eeckhoudt, L. Menegatti, M. (2016) Changing risks and optimal effort. Journal of Economic Behavior Organization 125, 97-106.

[5] Crainich, D., Eeckhoudt, L., Trannoy, A. (2013) Even (mixed) risk lovers are prudent. American Economic Review 103, 1529-1535.

[6] Di Crescenzo, A. (1999) A probabilistic analogue of the mean value theorem and its applications to reliability theory, Journal of Applied Probability 36, 709-716.

[7] Dionne, G., Eeckhoudt, L., (1985) Self-insurance, self-protection and increased risk aversion. Economics Letters 17, 39-42. 
[8] Dixit, A. (1987) Strategic behavior in contests. American Economic Review 77, 891-898.

[9] Eeckhoudt, L., Schlesinger H. (2006) Putting risk in its proper place. American Economic Review 96, 280-289.

[10] Ehrlich, L., Becker, G. (1972) Market insurance, self insurance and self protection. Journal of Political Economy 80, 623-648.

[11] Konrad, K. A., Schlesinger, H. (1997) Risk aversion in rent-seeking and rent-augmenting games. Economic Journal 107, 1671-1683.

[12] Hofmann, A., Peter, R. (2016) Self-Insurance, Self-Protection, and Saving: On Consumption Smoothing and Risk Management. Journal of Risk and Insurance 83(3), 719-734.

[13] Liu, L., Meyer, J., Rettenmaier A. J., Saving, T. R. (2018) Risk and risk aversion effects in contests with contingent payments Journal of Risk and Uncertainty 56, 289305.

[14] Menegatti, M. (2015) New resuts on high-order risk changes. European Journal of Operational Research 243, 678-681.

[15] Pratt, J. W. (1964) Risk Aversion in the Small and in the Large. Econometrica 32, $122-136$.

[16] Rothschild, M., Stiglitz J. E. (1970) Increasing Risk I: A Definition Journal of Economic Theory 2, 225-243

[17] Snow, A.(2011) Ambiguity aversion and the propensities for self-insurance and selfprotection Snow, Journal of Risk and Uncertainty 42, 27-43

[18] Treich, N. (2010) Risk-aversion and prudence in rent-seeking games. Public Choice $145,339-349$.

[19] Tullock, G. (1980) Efficient rent-seeking. In J. M. Buchanan, R. D. Tollison, G. Tullock (Eds.), Toward a theory of the rent-seeking society (pp. 97-112), Texas A. M. University Press, College Station. 